\title{
Hydrogen inhalation alleviates nonalcoholic fatty liver disease in metabolic syndrome rats
}

\author{
BOYAN LIU ${ }^{1,2^{*}}$, JUNLI XUE ${ }^{1,2^{*}}$, MENGYU ZHANG ${ }^{1,2}$, MINGYUE WANG $^{1,2}$, \\ TINGTING MA ${ }^{1,2}$, MIN ZHAO ${ }^{1,2}$, QIANQIAN GU ${ }^{1,2}$ and SHUCUN QIN ${ }^{1,2}$ \\ ${ }^{1}$ Taishan Institute for Hydrogen Biomedicine and ${ }^{2}$ Key Laboratory of Atherosclerosis in Universities of Shandong Province, \\ Institute of Atherosclerosis, Shandong First Medical University and Shandong Academy of Medical Sciences, \\ Tai'an, Shandong 271000, P.R. China
}

Received January 18, 2020; Accepted June 11, 2020

DOI: $10.3892 / \mathrm{mmr} .2020 .11364$

\begin{abstract}
Hydrogen exhibits therapeutic and preventive effects against various diseases. The present study investigated the potential protective effect and dose-dependent manner of hydrogen inhalation on high fat and fructose diet (HFFD)-induced nonalcoholic fatty liver disease (NAFLD) in Sprague-Dawley rats. Rats were randomly divided into four groups: i) Control group, regular diet/air inhalation; ii) model group, HFFD/air inhalation; iii) low hydrogen group, HFFD/4\% hydrogen inhalation; and iv) high hydrogen group, HFFD/67\% hydrogen inhalation. After a 10-week experiment, hydrogen inhalation ameliorated weight gain, abdominal fat index, liver index and body mass index of rats fed with HFFD and lowered the total area under the curve in an oral glucose tolerance test. Hydrogen inhalation also ameliorated the increase in liver lipid content and alanine transaminase and aspartate transaminase activities. Liver histopathologic changes evaluated with hematoxylin and eosin as well as Oil Red O staining revealed lower lipid deposition in hydrogen inhalation groups, consistent with the decrease in the expression of the lipid synthesis gene SREBP-1c. The majority of the indicators were affected following treatment with hydrogen in a dose-dependent manner. In conclusion, hydrogen inhalation may play a protective role by influencing the general state, lipid metabolism parameters, liver histology and liver function indicators in the rat model of metabolic syndrome with NAFLD.
\end{abstract}

Correspondence to: Professor Shucun Qin, Key Laboratory of Atherosclerosis in Universities of Shandong Province, Institute of Atherosclerosis, Shandong First Medical University and Shandong Academy of Medical Sciences, 2 Yingsheng East Road, Tai'an, Shandong 271000, P.R. China

E-mail: scqin@sdfmu.edu.cn

*Contributed equally

Key words: molecular hydrogen, metabolic syndrome, nonalcoholic fatty liver disease, lipid metabolism

\section{Introduction}

Metabolic syndrome (MS) is a cluster of metabolic derangements that may increase the risk of development of diabetes and cardiovascular diseases (1). In general, MS includes abdominal obesity, dyslipidemia, impaired fasting glucose level and high blood pressure (2,3). The prevalence of MS is increasing worldwide and, according to data from the International Diabetes Federation, about one-quarter of the world's adult population is suffering from it (4).

Nonalcoholic fatty liver disease (NAFLD) is one of the most common types of chronic liver diseases (5), characterized by a wide range of histopathological states ranging from simple steatosis to more severe fibrosis and cirrhosis, which may lead to liver failure or hepatocellular carcinoma (6). Hepatic morphology and functions may be adversely affected by MS, consequently leading to the development of NAFLD (7). NAFLD is also suggested to be a strong determinant of the development of MS (8). The prevalence of NAFLD has been steadily increasing along with metabolic conditions (9). The prevalence of MS with NAFLD is known to increase with a body mass index (BMI) of $18 \%$ in normal-weight NAFLD subjects to $67 \%$ in obese subjects (7).

The exact mechanisms underlying NAFLD have yet to be elucidated. Insulin resistance and chronic oxidative stress are reported to play major roles and serve as the first pathophysiological drivers of liver damage and NAFLD $(10,11)$. The histological hallmark of NAFLD is the accumulation of triacylglycerol (TG)-rich lipid droplets within hepatocytes (12). In addition, several abnormalities in various lipid classes have been identified in NAFLD (13). In over-nutrition, adipose tissue insulin resistance leads to inappropriate lipolysis and release of fatty acids into the circulation, which can be taken up and induce an overload in the liver (14). The excess fatty acids and glucose in the blood can be diverted to the oxidative pathways in other tissues and trigger oxidative stress including mitochondrial reactive oxygen species production and lipotoxic lipids (14). Nuclear factor $\kappa \beta(N F-\kappa B)$ signal also increases and then induces the production of pro-inflammatory mediators including tumor necrosis factor $\alpha$ (TNF- $\alpha$ ), interleukin (IL) 6 and IL-1 $\beta$ (15). 
At present, the standard of care for patients with NAFLD and MS focuses on lifestyle modification, including diet and physical activity targeting visceral adiposity $(16,17)$. Diverse medications have also been developed in an attempt to treat NAFLD. Vitamin $E$ has been used for its antioxidant ability to normalize liver enzymes or improve inflammation and hepatocyte ballooning $(18,19)$. Pioglitazone, an insulin sensitizer peroxisome proliferator-activated receptor $\gamma(\operatorname{PPAR} \gamma)$ agonist, is known to be effective in alleviating NAFLD. The American Association for Study of Liver Disease usually recommends the combined use of vitamin $\mathrm{E}$ and pioglitazone (20). Although insulin sensitizers such as pioglitazone and the antioxidant vitamin $\mathrm{E}$ have shown promising results, there are some concerns regarding adverse effects and long-term safety. Pioglitazone may cause weight gain, edema, osteoporosis and heart failure $(21,22)$, while vitamin $\mathrm{E}$ is considered to be fairly benign. Vitamin $\mathrm{E}$ is fat-soluble and could exert adverse effects at high doses $(23,24)$. Therefore, it is of considerable interest to explore safe treatment regimens for NAFLD and MS.

Molecular hydrogen $\left(\mathrm{H}_{2}\right)$ is the smallest gas molecule with a strong ability to penetrate and access cells and even organelles (25). $\mathrm{H}_{2}$ has been regarded as biochemically inert for a long time and little attention has been paid to its biological effect, although it was shown to inhibit the growth of squamous carcinoma in mice in 1975 (26). In 2007, Ohsawa et al (27) discovered the selective antioxidant mechanism of low concentration $\mathrm{H}_{2}(<4 \%$, v/v) for ischemia/reperfusion injury. In recent years, several studies have confirmed its protective effects against various diseases, including central nervous system pathology, cardiovascular and cerebrovascular diseases, cancer, organ injuries and dermatologic diseases. Our previous studies have demonstrated the safety and effectiveness of $\mathrm{H}_{2}$ in metabolic disorders, especially atherosclerosis, through animal and clinical experiments (28-31). However, $\mathrm{H}_{2}$ inhalation has never been tried in alleviating NAFLD.

The present study assessed the potential beneficial effects of $\mathrm{H}_{2}$ on the general state, lipid metabolism parameters and liver histology and functionality in a rat model of MS induced by high fat and fructose diet (HFFD).

\section{Materials and methods}

Animals and model establishment. A total of 46 male Sprague-Dawley rats (age, 6 weeks old; weight, 240-260 g) were purchased from the Beijing Vital River Laboratory Animal Technology Co., Ltd. The rats were housed under standard conditions with suitable temperature $\left(22 \pm 1^{\circ} \mathrm{C}\right)$, humidity (50-60\%) and a 12-h light/dark cycle. Animals were provided with food and water ad libitum. All the experiments were conducted following the guidelines of the laboratory animal ethics committee of Shandong First Medical University and Shandong Academy of Medical Sciences (http://marxism. sdfmu.edu.cn/info/1126/1104.htm, version 2011). After one week of acclimatization, the rats were randomly allocated to four different experimental groups ( $\mathrm{n}=10-12$ /group) as follows: i) Control group (Con) exposed to a regular diet and air; ii) model group (HFFD) exposed to HFFD and air; iii) low $\mathrm{H}_{2}$ group (HFFD $+\mathrm{LH}_{2}$ ) exposed to HFFD and $4 \% \mathrm{H}_{2}$; and, iv) high $\mathrm{H}_{2}$ group ( $\mathrm{HFFD}+\mathrm{HH}_{2}$ ) exposed to HFFD and $67 \% \mathrm{H}_{2}$.
The model was induced by HFFD feeding. The diet contained $24.2 \%$ protein, $42.1 \%$ carbohydrates and $25.4 \%$ fat, providing 19.8, 35.2 and $45 \%$ calories, respectively. In addition, the diet contained $2 \%$ cholesterol and $20 \%$ fructose. The animals began HFFD feeding after a one-week acclimation period until the end of the experiment. The experiment lasted for 10 weeks.

$\mathrm{H}_{2}$ inhalation. Low (4\%) and high (67\%) concentrations of $\mathrm{H}_{2}$ were provided by our self-developed device. $\mathrm{H}_{2}$ and oxygen were supplied from cylinders, while the air was provided by an air generator. The flow rate of each gas was adjusted by a flow meter. The 3 gases were mixed in a plastic box and pumped into a sealed animal chamber with a total flow rate of $3 \mathrm{l} / \mathrm{min}$ for $2 \mathrm{~h}$ once daily. Gas detectors XP-3140 (New Cosmos Electric Co., Ltd.) and JR2000-O2 (JingRuiBo Technology Co., Ltd.) were used to monitor the concentrations of $\mathrm{H}_{2}$ and oxygen (21\%) to confirm the stability of each gas component. The gas intervention started on the day of HFFD feeding and continued until the end of the experiment.

Sampling. Food intake was recorded every other day in the last week. After 10 weeks of the experiment, the rats were anesthetized by an intraperitoneal injection of chloral hydrate (400 mg/kg body weight) and body weight, abdominal circumference and tibial length were measured. Blood samples (8-10 $\mathrm{ml})$ were collected from the inferior vena cava, centrifuged at $3,000 \times \mathrm{g}$ for $15 \mathrm{~min}$ at $4^{\circ} \mathrm{C}$ and stored at $-80^{\circ} \mathrm{C}$ for further biochemical analyses. Rats were sacrificed by cardiac perfusion using phosphate-buffered saline (PBS) without fixation. Animal death was defined as mydriasis, respiratory arrest and cardiac arrest for a period of $>5 \mathrm{~min}$. Liver and abdominal white fats (including perirenal, perigonadal and mesenteric fats) were dissected and weighed. Parts of the tissues were fixed in $4 \%$ paraformaldehyde for $24 \mathrm{~h}$ at room temperature for histopathological examination, while the remaining tissues were immediately sliced, frozen under liquid nitrogen and stored at $-80^{\circ} \mathrm{C}$ for the evaluation of biochemical parameters. Liver and abdominal fat indices were calculated by dividing the tissue weight $(\mathrm{g})$ by tibial length $(\mathrm{cm})$.

Oral glucose tolerance test (OGTT). OGTT was performed during the last week of the experiment. Rats were deprived of their respective diets overnight and then administered with $2 \mathrm{~g}$ glucose $/ \mathrm{kg}$ body weight by oral gavage. Blood samples were collected from the tail vein at $0,30,60,90$ and 120 min after oral glucose loading and blood glucose levels were measured with a calibrated OneTouch UltraEasy glucometer (LifeScan). The total area under the curve (AUC) for OGTT was calculated by the trapezoid method.

Quantification of plasma biochemical markers. Biochemical parameters indicative of lipid levels (total cholesterol and total triglycerides) and liver functions [alanine aminotransferase (ALT), aspartate aminotransferase (AST) and lactic dehydrogenase ( $\mathrm{LDH})$ ] were measured with an automatic biochemistry analyzer Chemray-240 (Rayto Life and Analytical Sciences Co., Ltd.).

Measurement of intrahepatic lipid content. Lipid extraction from the liver was carried out using the methyl-tert-butyl 
ether (MTBE) method with some modifications. Briefly, liver tissues $(8-10 \mathrm{mg})$ were homogenized in $280 \mu 1$ of cold methanol containing an internal standard mixture [cholesteryl ester (CE) 17:0, TG 17:0/17:1/17:0 and d7-cholesterol]. In total, $50 \mu 1$ of homogenates were stored to analyze protein content using a commercial bicinchoninic acid protein assay kit (Beijing Solarbio Science \& Technology Co., Ltd.). MTBE $(1 \mathrm{ml})$ was added to the remaining volume of homogenates; the samples were vortexed and transferred to a rotary spinner at $40 \mathrm{rpm}$ for $1 \mathrm{~h}$ at room temperature. Phase separation was induced by adding $325 \mu \mathrm{l}$ water. After vortexing, the samples were centrifuged at $10,000 \mathrm{xg}$ for $10 \mathrm{~min}$ at $4^{\circ} \mathrm{C}$. The upper hydrophobic fraction $(350 \mu \mathrm{l})$ was transferred to another tube, dried with $\mathrm{N}_{2}$ gas and stored at $-80^{\circ} \mathrm{C}$. The lipid extracts were reconstituted in $200 \mu \mathrm{l}$ of acetonitrile:2-propanol $(1: 1, \mathrm{v} / \mathrm{v})$ prior to liquid chromatography tandem mass spectrometry (LC-MS/MS) analysis.

LC-MS/MS was performed using a Shimadzu LC-20 AD binary pump system coupled to a SIL-20AC autoinjector and interfaced with an ABI 4000 QTrap mass spectrometer (SCIEX). Chromatographic separations were carried out on a Waters Symmetry C18 column $(3.5 \mu \mathrm{m}, 2.1 \mathrm{~mm}$ i.d.x100 mm) with a Waters $\mathrm{C} 18$ guard column $(3.5 \mu \mathrm{m}, 2.1 \mathrm{~mm}$ i.d.x10 mm) at $40^{\circ} \mathrm{C}$ (Waters Corporation). The injection volume was $10 \mu \mathrm{l}$, while the flow rate was $0.3 \mathrm{ml} / \mathrm{min}$. The mobile phase comprised (A) $10 \mathrm{mM}$ ammonium formate in acetonitrile: Water: Formic acid (83:17:0.1, v/v/v) and (B) $10 \mathrm{mM}$ ammonium formate in acetonitrile: 2-propanol: formic acid (50:50:0.1, v/v/v). Isocratic elution was performed with $95 \%$ B for 16 min.

Detection was accomplished at the multiple reaction monitoring mode with positive-ion detection. For CE and TG, electrospray ionization source was selected with the following settings: Ion spray voltage $=5,500 \mathrm{~V}$; ion source heater temperature $=400^{\circ} \mathrm{C}$; source gas $1=40 \mathrm{psi}$; source gas $2=40 \mathrm{psi}$; and curtain gas $=10$ psi. For free cholesterol, atmospheric pressure chemical ionization source was selected with the following parameters: Nebulizer gas pressure of $55 \mathrm{psi}$; curtain gas pressure of $20 \mathrm{psi}$; nebulizer current of $3 \mu \mathrm{A}$, source temperature of $550^{\circ} \mathrm{C}$ and medium nitrogen collision gas pressure.

The precursor-to-product ion $\mathrm{m} / \mathrm{z}$ transitions, declustering potentials and collision energy are summarized in Table SI . Relative quantification of lipids in samples was carried out based on the intensity of each species divided by the intensity of the internal standards and protein concentrations.

Liver histology. Fixed tissues were washed with pure water for $12 \mathrm{~h}$, dehydrated in gradient ethyl alcohol concentrations and embedded in paraffin. Sections $(5 \mu \mathrm{m})$ were cut, deparaffinized, hydrated and stained with hematoxylin at room temperature for $5 \mathrm{~min}$, followed by $1 \%$ eosin staining at room temperature for $3 \min (\mathrm{H} \& \mathrm{E})$.

To assess lipid deposition, liver tissues were frozen in liquid nitrogen, embedded in optimum cutting temperature compound, sectioned at $8 \mu \mathrm{m}$ thickness and stained with $0.5 \%$ Oil Red $\mathrm{O}$ for $15 \mathrm{~min}$ at $60^{\circ} \mathrm{C}$. All the slides were viewed and images captured using an Olympus BX53 light microscope (Olympus Corporation) at magnification, $\mathrm{x} 400$.

Reverse transcription-quantitative (RT-q) PCR. Liver tissues were homogenized in cold TRIzol ${ }^{\circledR}$ reagent (Thermo Fisher
Scientific, Inc.) using a high-speed tissue grinding instrument (KZ-II, Wuhan Servicebio Technology Co., Ltd.). RNA was extracted following the manufacturer's instructions (Invitrogen; Thermo Fisher Scientific, Inc.) and its purity and concentration were measured using an Ultraviolet-visible spectrophotometer (DS-11, DeNovix Inc.). Total RNA was reverse transcribed into first-strand cDNA using HiFiScript gDNA Removal RT MasterMix (CoWin Biosciences) at $37^{\circ} \mathrm{C}$ for $15 \mathrm{~min}$. For RT-qPCR analysis, the reaction was carried out using UltraSYBR Mixture in a total volume of $25 \mu \mathrm{l}$ prepared in accordance with the instruction of the reagent kit (CoWin Biosciences). Expression of SREBP- $1 c$ gene was measured and $\beta$-actin was used as a housekeeping gene. The primer sequences were as follows: SREBP-1c, 5'-GCAACACTGGCA GAGATCTACGT-3' (forward) and 5'-TGGCGGGCACTA CTTAGGAA-3' (reverse); $\beta$-actin, 5 '-TTCCTTCCTGGGTAT GGAAT-3' (forward) and 5'-GAGGAGCAATGATCTTGA TC-3' (reverse). RT-qPCR reactions were performed under the following conditions: $95^{\circ} \mathrm{C}$ for $10 \mathrm{~min}$, followed by 40 cycles of $95^{\circ} \mathrm{C}$ for $10 \mathrm{sec}, 61^{\circ} \mathrm{C}$ for $32 \mathrm{sec}$ and $72^{\circ} \mathrm{C}$ for $32 \mathrm{sec}$. The $2^{-\Delta \Delta \mathrm{Ca}}$ method (32) was used to normalize mRNA expression level to that of $\beta$-actin. This experiment was repeated three times independently.

Statistical analysis. Results are presented as mean \pm standard deviation. Shapiro-Wilk normality test $(\alpha=0.05)$ was used to determine whether sample group data were distributed normally. For parametric data, one-way analysis of variance was performed followed by the least significant difference (LSD) post hoc test. For non-parametric data, Kruskal-Wallis tests were performed. All statistical analyses were performed using SPSS 20.0 (IBM Corp.) for Windows. P $<0.05$ was considered to indicate a statistically significant difference.

\section{Results}

$\mathrm{H}_{2}$ inhalation ameliorated body weight gain and compositional changes. Although the mean daily food intake was reduced $(\mathrm{P}<0.05$, Fig. 1A) in HFFD-fed rats, their body weight, BMI, abdominal fat index and liver index were significantly higher compared with those of normal diet-fed rats. Low and high concentrations of $\mathrm{H}_{2}$ inhalation could reduce these parameters in a dose-dependent manner (Fig. 1B-E). Significant differences of BMI were observed between HFFD and high $\mathrm{H}_{2}$ $\left(\mathrm{HFFD}+\mathrm{HH}_{2}\right)$ groups $(\mathrm{P}<0.01)$. No difference in kidney index was detected among different groups (Fig. 1F).

$\mathrm{H}_{2}$ inhalation increased the AUC for OGTT. OGTT results revealed that the plasma glucose level of all rats increased after oral glucose administration. Rats from the HFFD group demonstrated the highest level of blood glucose. Following $\mathrm{H}_{2}$ inhalation, the values significantly decreased in a dose-dependent manner (Fig. 2A). The area under the curve (AUC) for OGTT was significantly increased for the HFFD-fed rats, which is indicative of the impairment in glucose tolerance $(\mathrm{P}<0.05$, Fig. $2 \mathrm{~B}) . \mathrm{H}_{2}$ inhalation at both low and high concentrations could lower the AUC for OGTT by 9.0 and $14.8 \%$, respectively (Fig. 2A and B). No significant differences were observed between the low and high $\mathrm{H}_{2}$ inhalation groups. 
A

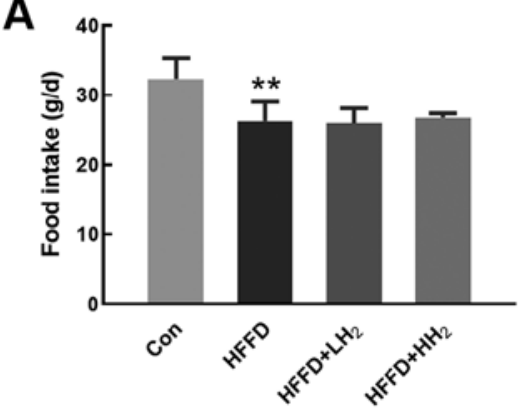

D

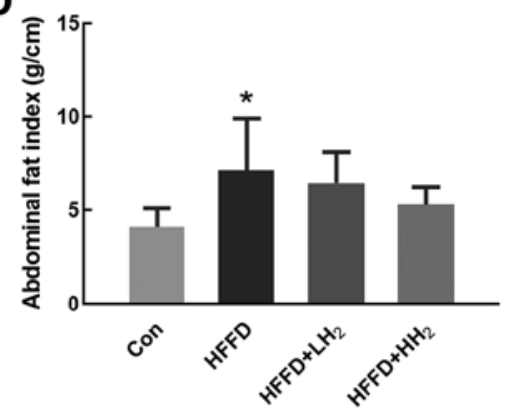

B

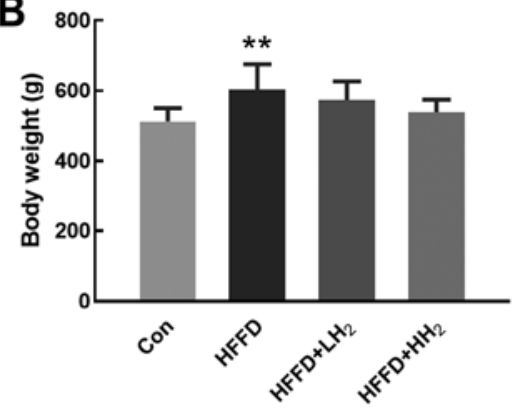

E

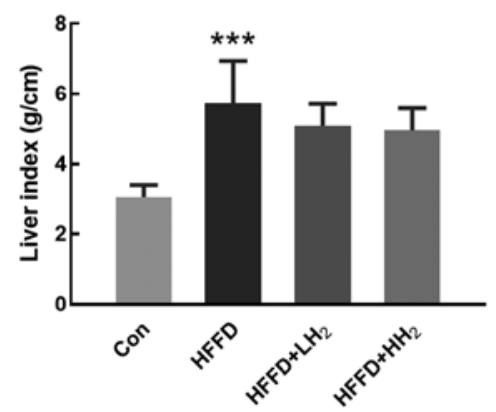

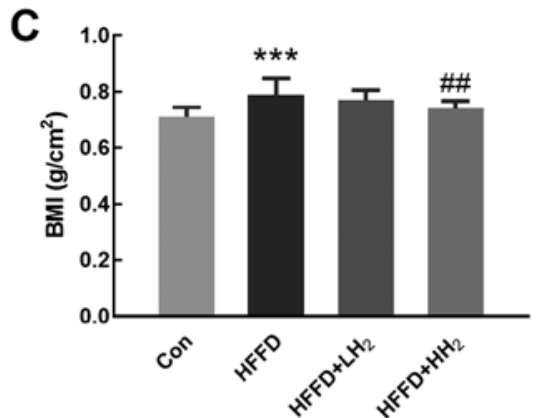

F

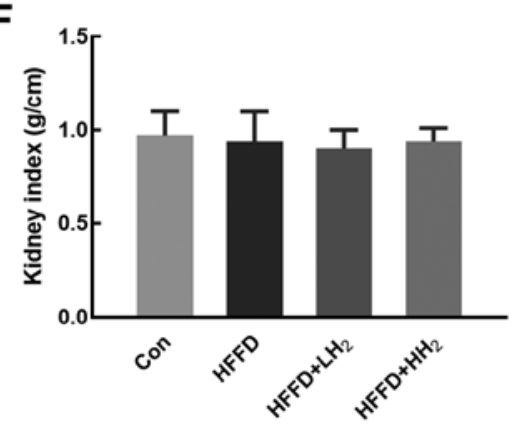

Figure 1. Effects of $\mathrm{H}_{2}$ inhalation on body weight gain and compositional changes. (A) Changes in food intake, (B) body weight, (C) BMI, (D) abdominal fat index, (E) liver index, and (F) kidney index of rats from Con (control group), HFFD (model group), $\mathrm{HFFD}+\mathrm{LH} \mathrm{H}_{2}$ and $\mathrm{HFFD}+\mathrm{HH} \mathrm{H}_{2}$ groups. ${ }^{*} \mathrm{P}<0.05,{ }^{* *} \mathrm{P}<0.01$ and ${ }^{* * *} \mathrm{P}<0.001$ vs. the Con group; ${ }^{\# \#} \mathrm{P}<0.01$ vs. the HFFD group. $\mathrm{n}=10-12$ per group. Con, control group; $\mathrm{HFFD}$, high fat and fructose diet group; $\mathrm{LH}_{2}$, low $\mathrm{H}_{2}$ group; $\mathrm{HH}_{2}$, high $\mathrm{H}_{2}$ group.

$\mathrm{H}_{2}$ inhalation improved plasma biomarkers. Plasma total triglyceride levels were significantly higher in the rats from HFFD groups compared with those from the Con group. $\mathrm{H}_{2}$ inhalation at both low and high concentrations could significantly prevent the increase in plasma total triglycerides $\left(\mathrm{P}<0.05\right.$, Fig. 2C). Rats fed with HFFD and subjected to $\mathrm{H}_{2}$ inhalation demonstrated no significant changes in plasma total cholesterol (Fig. 2D). In comparison with the rats from the Con group, those from the HFFD feeding group had significantly increased plasma activities for ALT, AST and LDH, the biomarkers of liver functions $(\mathrm{P}<0.05, \mathrm{P}<0.001$ and $\mathrm{P}<0.01$, respectively). Rats from the $\mathrm{HFFD}+\mathrm{LH}_{2}$ group had lower plasma ALT, AST and LDH activities compared with the HFFD group (Fig. 2E-G). A low concentration of $\mathrm{H}_{2}$ seemed to exert better effects than a high concentration.

$\mathrm{H}_{2}$ inhalation regulated hepatic fat accumulation. HFFD consumption led to the development of fatty liver. Hepatic lipid profiles for cholesterol and TG species were analyzed and are presented in Fig. 3. In comparison with the control rats, those fed with HFFD demonstrated a significant increase in the sum of hepatic cholesterol and TG species (16.4- and 6.0-fold, respectively). After inhalation of low concentration of $\mathrm{H}_{2}$, the total hepatic cholesterol and TG levels were suppressed by 11.1 and $26.2 \%$, respectively. Inhalation of a high concentration of $\mathrm{H}_{2}$ resulted in a significant decrease in the levels of total hepatic cholesterol and TG by 32.2 and $40.5 \%$, respectively $(\mathrm{P}<0.05$, Fig. 3). The relative fold increase in different cholesterol and TG species demonstrated great variations. The contents of most species decreased after $\mathrm{H}_{2}$ inhalation.

$\mathrm{H}_{2}$ inhalation regulated hepatic fat accumulation. Evaluation of the general morphology of the liver tissue from Con rats revealed a uniform color and texture. The volume of the liver tissue seemed to slightly increase for the rats from HFFD group, consistent with the observations such as round blunt edges, loose texture, light beige color and greasy feeling with dense small holes. $\mathrm{H}_{2}$ intervention could partly alleviate these phenomena (Fig. S1). After H\&E staining, the hepatocytes from the Con group were normal and distinct, regularly arranged and formed clear and complete hepatic cords (Fig. 4A). Only few tiny lipid droplets were observed after Oil Red O staining (Fig. 4E). By contrast, the tissue from the HFFD group demonstrated round fat vacuoles (lipid droplets) of different sizes in the cytoplasm and fuzzy or even broken intercellular boundaries. In addition, the hepatic cord demonstrated disordered arrangements (Fig. 4B and F). This condition was alleviated following $\mathrm{H}_{2}$ treatment, especially at high concentration (Fig. 4C and D). Smaller and less red-dyed fatty droplets were observed after intervention with low and high concentrations of $\mathrm{H}_{2}$ (Fig. $4 \mathrm{G}$ and $\mathrm{H}$ ). These findings are in agreement with the changes in the hepatic cholesterol and TG contents (Fig. 3). The expression of the lipid synthesis gene SREBP-1c was significantly upregulated in HFFD rats $(\mathrm{P}<0.001$, Fig. 4I). Inhalation of low and high concentrations of $\mathrm{H}_{2}$ significantly decreased SREBP-1c mRNA expression in a dose-dependent manner $(\mathrm{P}<0.05$ and $\mathrm{P}<0.01$, respectively; Fig. 4I).

\section{Discussion}

As a small molecular gas, $\mathrm{H}_{2}$ may diffuse into the target tissues without any hindrance. $\mathrm{H}_{2}$ administration can be achieved through inhalation, drinking $\mathrm{H}_{2}$-rich water, or injection of $\mathrm{H}_{2}$-rich saline (25). The beneficial effects of the inhalation of low concentrations (2 and 4\%) of $\mathrm{H}_{2}$ gas against ischemia/reperfusion injuries were first described 
A
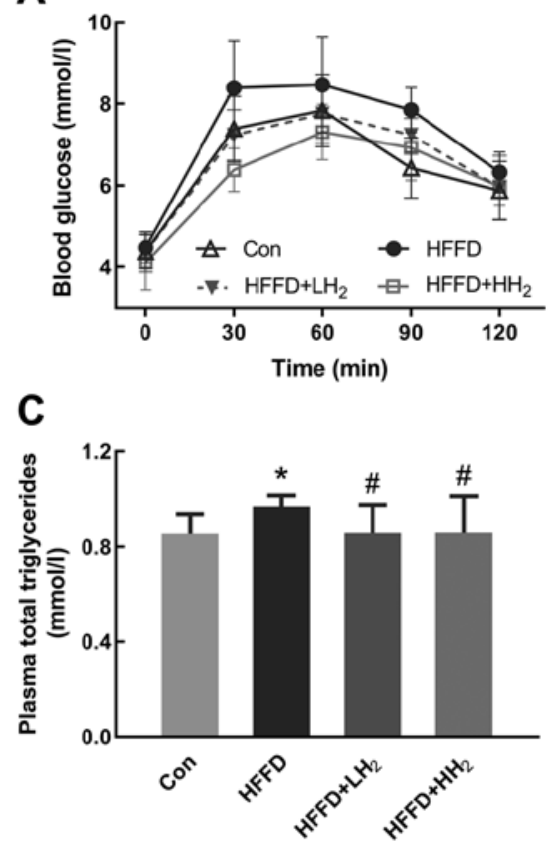

B
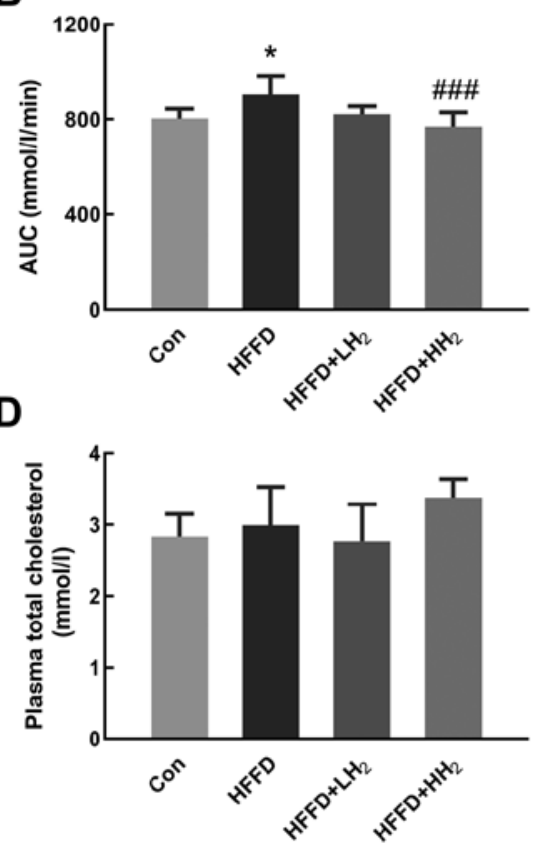

E

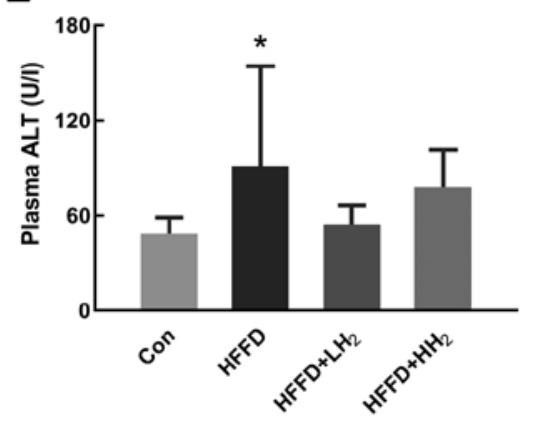

$\mathbf{F}$

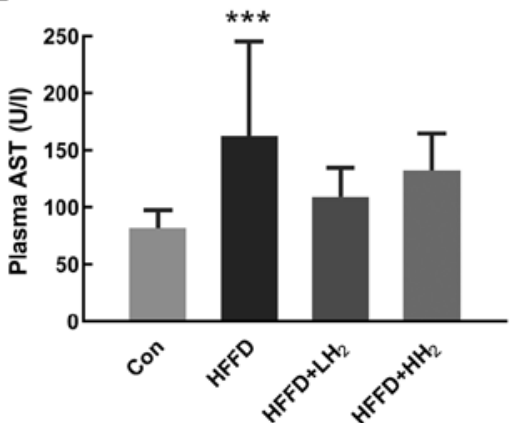

G

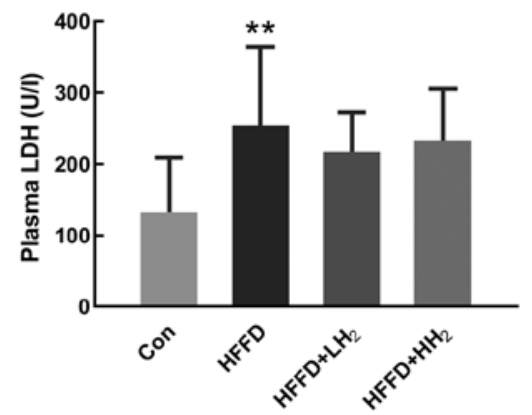

Figure 2. Effects of $\mathrm{H}_{2}$ inhalation on OGTT and plasma biochemical values. (A) Curve of OGTT and (B) AUC of OGTT (n=10 per group). Concentrations of (C) plasma total triglycerides, (D) total cholesterol, (E) ALT, (F) AST and (G) LDH (n=8-12 per group). Data are shown as mean \pm standard deviation. " $\mathrm{P}<0.05$, ${ }^{* *} \mathrm{P}<0.01$ and ${ }^{* * *} \mathrm{P}<0.001$ vs. the Con group; ${ }^{~} \mathrm{P}<0.05$ and ${ }^{\# \# "} \mathrm{P}<0.001$ vs. the HFFD group. OGTT, Oral glucose tolerance test; AUC, area under the curve; ALT, aminotransferase; AST, aspartate aminotransferase; $\mathrm{LDH}$, lactic dehydrogenase; Con, control group; HFFD, high fat and fructose diet group; $\mathrm{LH}_{2}$, low $\mathrm{H}_{2}$ group; $\mathrm{HH}_{2}$, high $\mathrm{H}_{2}$ group.

by Ohsawa et al (27). Thereafter, many studies have demonstrated the therapeutic effects of low concentrations of $\mathrm{H}_{2}$ inhalation on different diseases (33-35). In comparison with other methods, inhalation of $\mathrm{H}_{2}$ gas could provide more $\mathrm{H}_{2}$, especially at high concentrations. The recent development of the $\mathrm{H}_{2}$ generator has led to a gradual increase in the number of studies using high concentrations of $\mathrm{H}_{2}$. Inhalation of high concentrations of $\mathrm{H}_{2}$ has been proved to ameliorate ischemia/reperfusion injury $(36,37)$, endometriosis (38) and glyoxylate-induced calcium oxalate deposition (39) in animal models. Commercially available machines for inhalation of high concentrations of $\mathrm{H}_{2}$ have also been used in patients (40). Although both low and high concentrations of $\mathrm{H}_{2}$ have been applied in experiments, a comparison of their therapeutic effects has not been made. Thus, 4 and $67 \% \mathrm{H}_{2}$ were chosen in this study to investigate the dosage effect on NAFLD in MS rats. As the oxygen concentration directly generated from the commercial machine was $33 \%$, our self-made device was used to maintain the oxygen level in the mixed gas to approximately $21 \%$ to avoid effects of high concentrations of oxygen.
The occurrence and development of MS are influenced by several factors, particularly diet. Fat and fructose have been used in combination to induce MS and a HFFD-induced rodent model is the best model to study human MS (41). Thus, HFFD was used in this study to mimic human diets associated with the development of MS.

MS is a cluster of pathological conditions related to obesity, insulin resistance and dyslipidemia (3). The present study demonstrated that 10 -week HFFD feeding could lead to MS in rats by causing abdominal obesity and glucose intolerance as well as by increasing liver damage marker levels and dyslipidemia. Previous studies have shown the beneficial role of drinking $\mathrm{H}_{2}$-rich water in potential patients with $\mathrm{MS}(28,42)$ or MS rat model SHR. Cg-Lepr ${ }^{\mathrm{cp}} / \mathrm{NDmcr}$ (SHR-cp) (43). However, the effects of $\mathrm{H}_{2}$ inhalation on HFFD-induced MS in rats remain to be elucidated.

The present study provided direct evidence that inhalation of $\mathrm{H}_{2}$ during the 10-week experimental period decreased the body weight, BMI and abdominal fat index in a dose-dependent manner in rats fed with HFFD, demonstrating the anti-obesity 

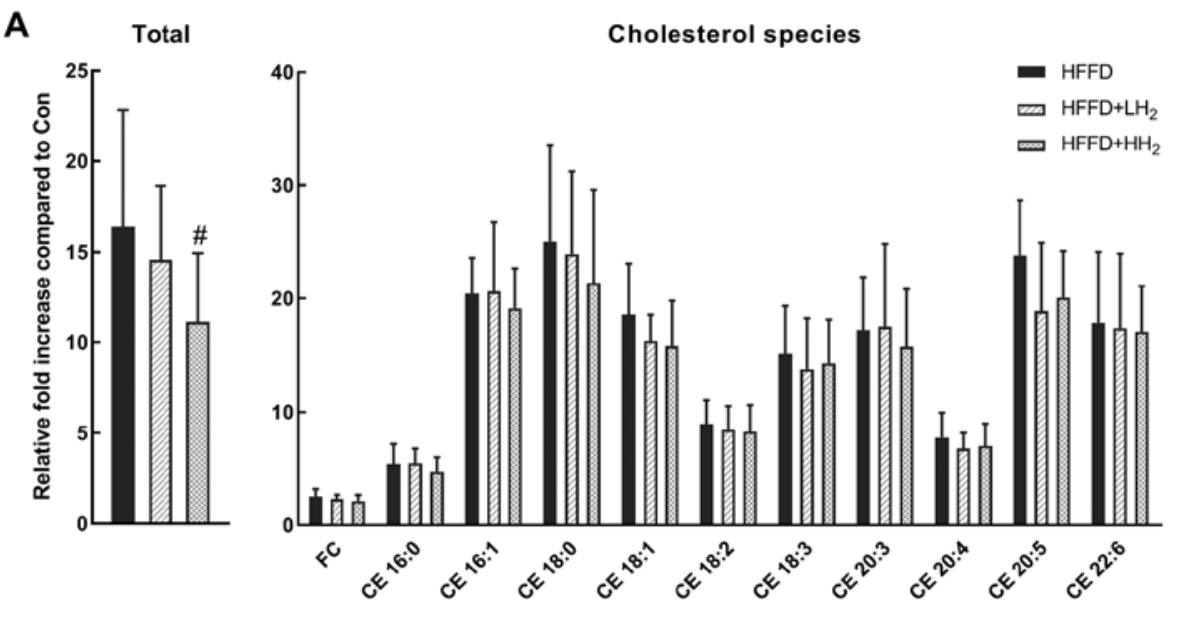

B
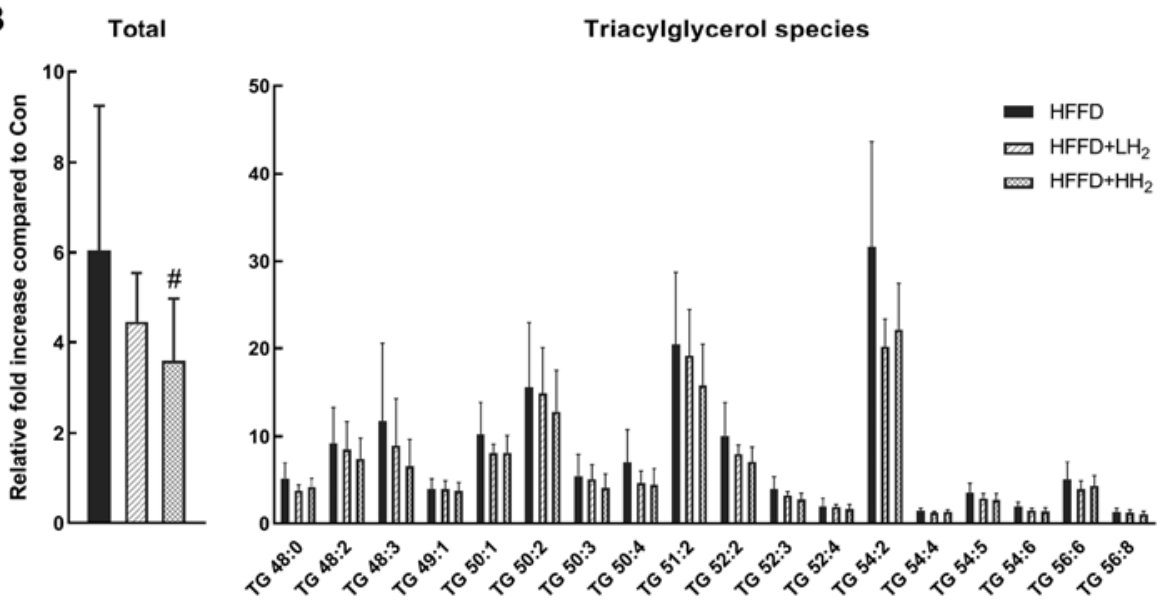

Figure 3. Total and different species of cholesterol and triglycerides in the liver tissues from different groups. (A) total and cholesterol species of HFFD, $\mathrm{HFFD}+\mathrm{LH}_{2}$ and HFFD $+\mathrm{HH}_{2}$ groups; (B) total and triacylglycerol species of HFFD, HFFD $+\mathrm{LH}_{2}$ and $\mathrm{HFFD}+\mathrm{HH}_{2}$ groups. ${ }^{\#} \mathrm{P}<0.05$ vs. the HFFD group. $\mathrm{n}=8-10$ per group. Con, control group; HFFD, high fat and fructose diet group; $\mathrm{LH}_{2}$, low $\mathrm{H}_{2}$ group; $\mathrm{HH}_{2}$, high $\mathrm{H}_{2}$ group; FC, free cholesterol; CE, cholesteryl ester; TG, triacylglycerol.

effect of $\mathrm{H}_{2}$, particularly at a high concentration. A previous clinical study demonstrated that the oral administration of $\mathrm{H}_{2}$-generating minerals can significantly reduce body fat percentage and arm fat index in middle-aged overweight women, which is indicative of the beneficial effects of $\mathrm{H}_{2}$ in the management of body composition in obesity (44). The present study confirmed the effects of $\mathrm{H}_{2}$ on visceral adipose depots and highlighted the beneficial effects of $\mathrm{H}_{2}$ against MS.

After challenging rats with a glucose load, both low and high concentrations of $\mathrm{H}_{2}$ could decrease the AUC values for OGTT. This observation is suggestive of the increase in glucose disposal, consistent with the results of previous findings that $\mathrm{H}_{2}$-rich saline improved glucose tolerance in a high-fat and high-sugar diet rat model after a single injection of streptozotocin (45). The results of the present study are suggestive of the increase in insulin sensitivity after treatment with $\mathrm{H}_{2}$ although no significant difference was observed between 4 and $67 \% \mathrm{H}_{2}$ inhalation. Therefore, different administration strategies and dosages of $\mathrm{H}_{2}$ play a positive role in the improvement of glucose tolerance. Plasma insulin level was also measured but no significant difference was observed (data not shown), which may due to limitations of the current animal model.
The liver is the most important tissue involved in the regulation of glucose and lipid metabolism (5). NAFLD is the most frequent liver disease commonly associated with MS (7). Intraperitoneal administration of $\mathrm{H}_{2}$-rich saline can improve NAFLD in rats (45). The present study investigated the effects of $\mathrm{H}_{2}$ inhalation on NAFLD in MS rats. The consumption of HFFD can lead to hepatic fat accumulation and liver dysfunctions (46), as is evident from the leakage of cellular enzymes such as ALT, AST and LDH (47). In the present study, the treatment with $\mathrm{H}_{2}$ in HFFD-fed rats could reduce the plasma levels of ALT, AST and LDH, although the differences were not significant. The statistical insignificance may be related to the insufficient number of animals in each group and the relatively short experimental period. It demonstrated better effects for plasma ALT and AST levels after low $\mathrm{H}_{2}$ treatment. The results suggested that inhalation of $\mathrm{H}_{2}$, especially at low concentrations, could attenuate hepatic necrosis by maintaining hepatocyte integrity. The mechanism underlying the enhanced effectiveness of low concentration of $\mathrm{H}_{2}$ requires further investigation.

Alterationsinhepaticlipidsareimportantpathophysiological hallmarks of fatty liver disease (12). In the present study, histological evaluation revealed the increase in fat vacuoles in 

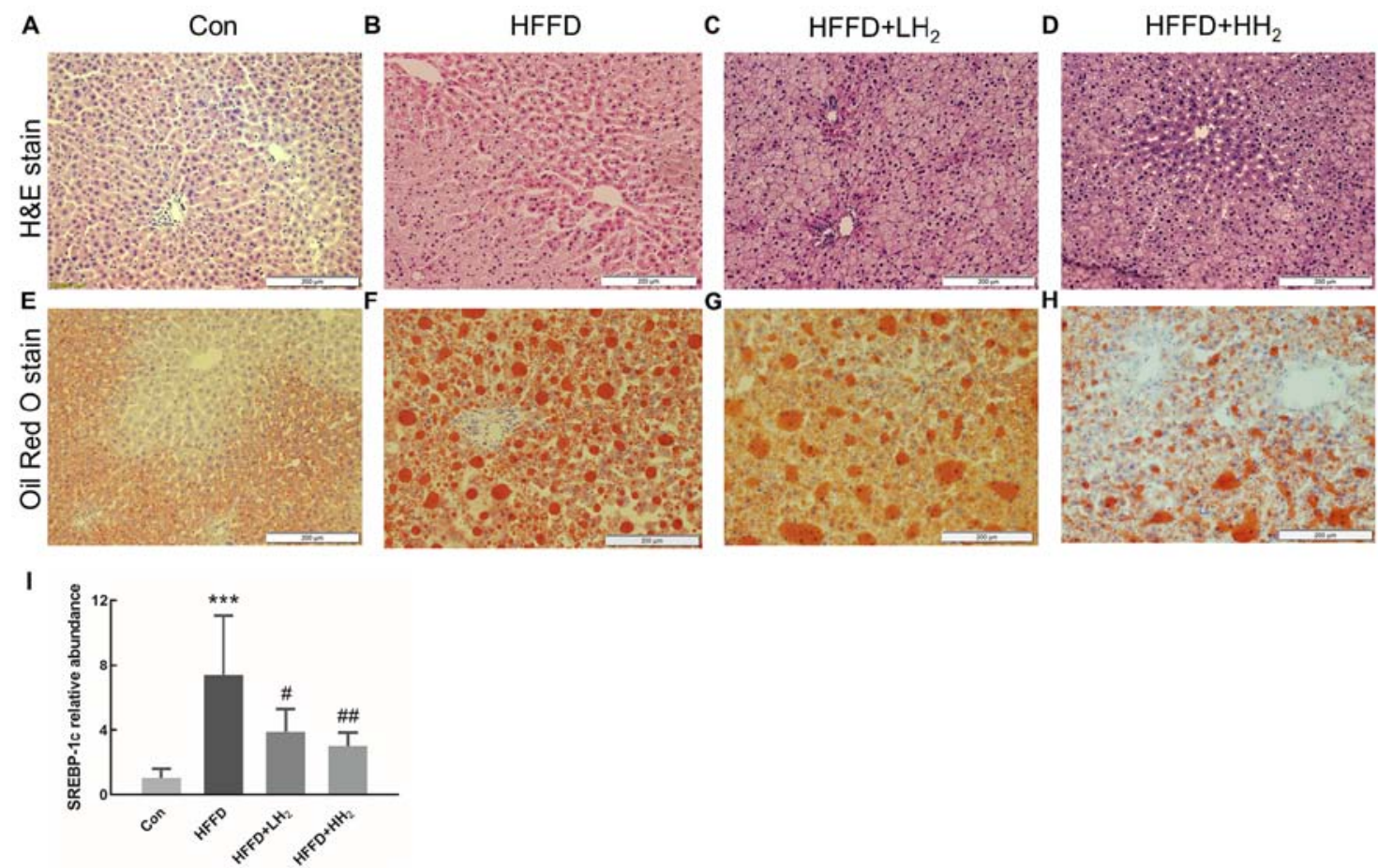

Figure 4. Pathological examination by H\&E staining and hepatic lipid accumulation analysis with Oil Red O staining in different groups. (A and E) Con, (B and F) HFFD group, (C and G) HFFD + $\mathrm{LH}_{2}$ Group, (D and H) HFFD $+\mathrm{HH}_{2}$ Group (n=10 per group; scale bars=200 $\mu$ m). (I) Hepatic mRNA expression of SREBP-1c ( $\mathrm{n}=4-6$ per group). ${ }^{* * * *} \mathrm{P}<0.001$ vs. the Con group; ${ }^{*} \mathrm{P}<0.05$ and ${ }^{\# \#} \mathrm{P}<0.01$ vs. the HFFD group. H\&E, hematoxylin and eosin; Con, control group; HFFD, high fat and fructose diet group; $\mathrm{LH}_{2}$, low $\mathrm{H}_{2}$ group; $\mathrm{HH}_{2}$, high $\mathrm{H}_{2}$ group.

the liver of rats fed with HFFD and this condition improved following $\mathrm{H}_{2}$ treatment in a dose-dependent manner. Rats fed with HFFD demonstrated a marked increase in all cholesterol species in the liver compared with those fed with the control diet. This observation is in line with a previously reported study, wherein the liver tissues from high fat, high cholesterol, cholate diet-fed mice demonstrated a significant increase in free cholesterol and different CE levels (48). Multiple defects in production, secretion and clearance of lipids in patients with NAFLD result in the accumulation of triglycerides in hepatocytes (12). A previous study reported that TG species are notably upregulated in the fatty liver of a genetically obese insulin-resistant ob/ob mouse model (49). In the present study, the liver from HFFD-fed rats demonstrated a marked increase in different triglyceride levels. Some triglycerides may serve as more specific biomarkers than total triglycerides. In obesity, the increase in triglycerides containing more saturated fatty acid moieties (e.g., TG 16:0/16:0/16:0) compared with those containing more unsaturated fatty acids (e.g., TG $18: 2 / 18: 2 / 18: 2)$ in the plasma is associated with an increase in BMI (50). Individual species could have distinct roles in the progression of NAFLD. Tu et al (48) reported a significant decrease in the levels of triglycerides with higher degrees of saturation (0-3 double bonds) compared with those with higher degrees of unsaturation ( $>3$ double bonds) in the liver of mice fed with a high fat, high cholesterol, cholate diet. In the present study, different triglycerides demonstrated varied levels of increment in HFFD group compared with those observed in the Con group. The increase in triglycerides with less unsaturated fatty acids was higher compared with those with polyunsaturated fatty acids. It is known that high-fat diet will increase saturated fatty acids such as palmitic acid and n- 6 unsaturated fatty acids such as arachidonic and then promote the inflammatory and oxidative responses $(51,52)$. In the present study, $\mathrm{H}_{2}$ treatment may alleviate the progress of NAFLD via changing the composition of fatty acids in liver lipids.

In a previous study, drinking $\mathrm{H}_{2}$-rich water was shown to slightly decrease the levels of hepatic cholesterol and triglycerides without any statistical significance in a methionine-choline-deficient diet-induced nonalcoholic steatohepatitis mouse model (53). The present study revealed the dose-dependent effect of $\mathrm{H}_{2}$ inhalation on the alleviation of liver lipid accumulation and $66 \% \mathrm{H}_{2}$ inhalation was found to significantly decrease hepatic cholesterol and triglycerides. Thus, inhalation of high concentrations of $\mathrm{H}_{2}$ may be a better choice for reducing liver lipid accumulation.

A previous study on human hepatoma HepG2 cells revealed that the mechanism underlying the effects of $\mathrm{H}_{2}$ gas on lipid metabolism disorders may involve the modulation of signal transduction pathways such as the c-Jun $\mathrm{N}$-terminal kinase pathway (54). Sterol regulatory element-binding proteins are a family of regulated transcription factors that stimulate lipid synthesis in the liver (55). SREBP-1c plays a unique role in the expression of the genes involved in hepatic triglyceride synthesis and may contribute to the pathogenesis of NAFLD (48). SREBP-1c in normal livers is low, but it shows an increase in liver steatosis and NAFLD (56). Recent findings have demonstrated that some agents can relieve metabolic disorders in the liver via SREBP-lc downregulation, PPAR- $\alpha$ upregulation and $N F-\kappa B$ inactivation (57). In the present study, $S R E B P-1 c$ mRNA increased in HFFD-fed rats, but decreased 
in $\mathrm{H}_{2}$ inhalation groups, which indicated the lipogenesis was inhibited. PPAR $\alpha$ is a key transcriptional regulator of fatty acid oxidation systems in the liver and is usually used together with SREBP-1c to estimate hepatic lipid homeostasis (57). Changes in the expression of SREBP-1c in the present study may be associated with PPAR- $\alpha$ expression (58). Meanwhile, the mRNA expression of fatty acid synthase $(F A S)$ was also examined, but no significant difference was achieved (data not shown). Further lipid synthesis- and lipolysis-related genes warrant further study.

In conclusion, the findings of the present study emphasized that $\mathrm{H}_{2}$ inhalation at both low and high concentrations could ameliorate the physical, metabolic and hepatic disorders in MS rats induced by HFFD. It reported a dosage effect of $\mathrm{H}_{2}$ on most indicators, except ALT, AST and LDH, and the lower concentration of $\mathrm{H}_{2}$ exerted better effects. The potential mechanism was inferred as the reduced synthesis of fatty acids and lipid accumulation in the liver. The results of the present study provided the basic data for clinical trials and $\mathrm{H}_{2}$ inhalation may be considered as a novel adjuvant for clinical treatment of NAFLD. However, the present study also has some limitations such as the lack of statistical significance for some factors and lack of detection of more genes associated with lipid metabolism. Further investigation is required to clarify the underlying mechanism and provide the basis for the selection of the best dose and concentration.

\section{Acknowledgements}

Not applicable.

\section{Funding}

The present study was supported by the National Natural Science Foundation of China (grant no. 81770855), Taishan Scholars Program of Shandong Province (grant no. ts201511057) and the Academic promotion programme of Shandong First Medical University (grant nos. 2019QL010 and 2019PT009).

\section{Availability of data and materials}

The analyzed data sets generated during the study are available from the corresponding author on reasonable request.

\section{Authors' contributions}

SQ was responsible for funding acquisition. Experiments were performed by BL, JX, MZ, MW, TM, MZ and QG. BL, $\mathrm{JX}$ and SQ conceived the methodology. BL and JX wrote the original draft of the manuscript and further writing, review and editing was by SQ. All authors read and approved the final manuscript.

\section{Ethics approval and consent to participate}

The ethics committee of the Shandong First Medical University and Shandong Academy of Medical Sciences approved and supervised the research proposal (approval no. 2017049).

\section{Patient consent for publication}

Not applicable.

\section{Competing interests}

The authors declare that they have no competing interests.

\section{References}

1. Kapravelou G, Martinez R, Andrade AM, Nebot E, Camiletti-Moiron D, Aparicio VA, Lopez-Jurado M, Aranda P, Arrebola F, Fernandez-Segura E, et al: Aerobic interval exercise improves parameters of nonalcoholic fatty liver disease (NAFLD) and other alterations of metabolic syndrome in obese Zucker rats. Appl Physiol Nutr Metab 40: 1242-1252, 2015.

2. Eckel RH, Grundy SM and Zimmet PZ: The metabolic syndrome. Lancet 365: 1415-1428, 2005

3. Eckel RH, Alberti KG, Grundy SM and Zimmet PZ: The metabolic syndrome. Lancet 375: 181-183, 2010.

4. Kaur J: A comprehensive review on metabolic syndrome. Cardiol Res Pract 2014: 943162, 2014.

5. Younossi ZM, Koenig AB, Abdelatif D, Fazel Y, Henry L and Wymer M: Global epidemiology of nonalcoholic fatty liver disease-meta-analytic assessment of prevalence, incidence, and outcomes. Hepatology 64: 73-84, 2016.

6. Bugianesi E, Leone N, Vanni E, Marchesini G, Brunello F, Carucci P, Musso A, De Paolis P, Capussotti L, Salizzoni M and Rizzetto M: Expanding the natural history of nonalcoholic steatohepatitis: From cryptogenic cirrhosis to hepatocellular carcinoma. Gastroenterology 123: 134-140, 2002.

7. Marchesini G, Bugianesi E, Forlani G, Cerrelli F, Lenzi M, Manini R, Natale S, Vanni E, Villanova N, Melchionda N and Rizzetto M: Nonalcoholic fatty liver, steatohepatitis, and the metabolic syndrome. Hepatology 37: 917-923, 2003.

8. Lonardo A, Ballestri S, Marchesini G, Angulo P and Loria P: Nonalcoholic fatty liver disease: A precursor of the metabolic syndrome. Dig Liver Dis 47: 181-190, 2015.

9. Younossi ZM, Stepanova M, Afendy M, Fang Y, Younossi Y, Mir $\mathrm{H}$ and Srishord M: Changes in the prevalence of the most common causes of chronic liver diseases in the United States from 1988 to 2008. Clin Gastroenterol Hepatol 9: 524-530.e1, 2011.

10. Sanyal AJ, Campbell-Sargent C, Mirshahi F, Rizzo WB, Contos MJ, Sterling RK, Luketic VA, Shiffman ML and Clore JN: Nonalcoholic steatohepatitis: association of insulin resistance and mitochondrial abnormalities. Gastroenterology 120 : 1183-1192, 2001.

11. Browning JD and Horton JD: Molecular mediators of hepatic steatosis and liver injury. J Clin Invest 114: 147-152, 2004.

12. Tang A, Desai A, Hamilton G, Wolfson T, Gamst A, Lam J, Clark L, Hooker J, Chavez T, Ang BD, et al: Accuracy of MR imaging-estimated proton density fat fraction for classification of dichotomized histologic steatosis grades in nonalcoholic fatty liver disease. Radiology 274: 416-425, 2015.

13. Sanyal AJ and Pacana T: A lipidomic readout of disease progression in a diet-induced mouse model of nonalcoholic fatty liver disease. Trans Am Clin Climatol Assoc 126: 271-288, 2015.

14. Tsatsoulis A, Mantzaris MD, Bellou S and Andrikoula M: Insulin resistance: An adaptive mechanism becomes maladaptive in the current environment-an evolutionary perspective. Metabolism 62: 622-633, 2013.

15. Valenzuela R and Videla LA: The importance of the long-chain polyunsaturated fatty acid $n-6 / n-3$ ratio in development of non-alcoholic fatty liver associated with obesity. Food Funct 2: 644-648, 2011.

16. Kim D, Touros A and Kim WR: Nonalcoholic fatty liver disease and metabolic syndrome. Clin Liver Dis 22: 133-140, 2018.

17. Chalasani N, Younossi Z, Lavine JE, Diehl AM, Brunt EM, Cusi K, Charlton $M$ and Sanyal AJ: The diagnosis and management of non-alcoholic fatty liver disease: Practice guideline by the American association for the study of liver diseases, American college of gastroenterology, and the american gastroenterological association. Hepatology 55: 2005-2023, 2012.

18. Hickman I and Macdonald G: Is vitamin E beneficial in chronic liver disease? Hepatology 46: 288-290, 2007. 
19. Sanyal AJ, Mofrad PS, Contos MJ, Sargeant C, Luketic VA, Sterling RK, Stravitz RT, Shiffman ML, Clore J and Mills AS: A pilot study of vitamin $E$ versus vitamin $E$ and pioglitazone for the treatment of nonalcoholic steatohepatitis. Clin Gastroenterol Hepatol 2: 1107-1115, 2004.

20. Chalasani N, Younossi Z, Lavine JE, Charlton M, Cusi K, Rinella M, Harrison SA, Brunt EM and Sanyal AJ: The diagnosis and management of nonalcoholic fatty liver disease: Practice guidance from the American association for the study of liver diseases. Hepatology 67: 328-357, 2018.

21. Juurlink DN, Gomes T, Lipscombe LL, Austin PC, Hux JE and Mamdani MM: Adverse cardiovascular events during treatment with pioglitazone and rosiglitazone: Population based cohort study. BMJ 339: b2942, 2009.

22. Hossain N, Kanwar P and Mohanty SR: A comprehensive updated review of pharmaceutical and nonpharmaceutical treatment for NAFLD. Gastroenterol Res Pract 2016: 7109270, 2016.

23. Gee PT: Unleashing the untold and misunderstood observations on vitamin E. Genes Nutr 6: 5-16, 2011.

24. Bjelakovic G, Nikolova D, Gluud LL, Simonetti RG and Gluud C: Mortality in randomized trials of antioxidant supplements for primary and secondary prevention: Systematic review and meta-analysis. JAMA 297: 842-857, 2007.

25. Nicolson GL, de Mattos GF, Settineri R, Costa C, Ellithorpe R, Rosenblatt S, La Valle J, Jimenez A and Ohta S: Clinical Effects of Hydrogen Administration: From Animal and Human Diseases to Exercise Medicine. Int J Clin Med 7: 32, 2016.

26. Dole M, Wilson FR and Fife WP: Hyperbaric hydrogen therapy: A possible treatment for cancer. Science 190: 152-154, 1975.

27. Ohsawa I, Ishikawa M, Takahashi K, Watanabe M, Nishimaki K, Yamagata K, Katsura K, Katayama Y, Asoh S and Ohta S: Hydrogen acts as a therapeutic antioxidant by selectively reducing cytotoxic oxygen radicals. Nat Med 13: 688-694, 2007.

28. Song G, Li M, Sang H, Zhang L, Li X, Yao S, Yu Y, Zong C, Xue Y and Qin S: Hydrogen-rich water decreases serum LDL-cholesterol levels and improves HDL function in patients with potential metabolic syndrome. J Lipid Res 54: 1884-1893, 2013.

29. Song G, Tian H, Qin S, Sun X, Yao S, Zong C, Luo Y, Liu J, Yu Y, Sang $H$ and Wang $X$ : Hydrogen decreases athero-susceptibility in apolipoprotein B-containing lipoproteins and aorta of apolipoprotein E knockout mice. Atherosclerosis 221: 55-65, 2012.

30. Zong C, Song G, Yao S, Li L, Yu Y, Feng L, Guo S, Luo T and Qin S: Administration of hydrogen-saturated saline decreases plasma low-density lipoprotein cholesterol levels and improves high-density lipoprotein function in high-fat diet-fed hamsters. Metabolism 61: 794-800, 2012

31. Song G, Zong C, Zhang Z, Yu Y, Yao S, Jiao P, Tian H, Zhai L, Zhao H, Tian S, et al: Molecular hydrogen stabilizes atherosclerotic plaque in low-density lipoprotein receptor-knockout mice. Free Radic Biol Med 87: 58-68, 2015

32. Livak KJ and Schmittgen TD: Analysis of relative gene expression data using real-time quantitative PCR and the 2(-Delta Delta C(T)) method. Methods 25: 402-408, 2001.

33. Choi K, Kim H, Hee S, Jin S and Yi H: Neuroprotective effects of hydrogen inhalation in an experimental rat intracerebral hemorrhage model. Brain Res Bull 142: 122-128, 2018.

34. Wang L, Zhao C, Wu S, Xiao G, Zhuge X, Lei P and Xie K: Hydrogen gas treatment improves the neurological outcome after traumatic brain injury via increasing miR-21 expression. Shock 50: 308-315, 2018

35. Zhou H, Fu Z, Wei Y, Liu J, Cui X, Yang W, Ding W, Pan P and Li W: Hydrogen inhalation decreases lung graft injury in brain-dead donor rats. J Heart Lung Transpl 32: 251-258, 2013.

36. Chen O, Cao Z, Li H, Ye Z, Zhang R, Zhang N, Huang J, Zhang T, Wang L, Han L, et al: High-concentration hydrogen protects mouse heart against ischemia/reperfusion injury through activation of the PI3K/Akt1 pathway. Sci Rep 7: 14871, 2017.

37. Li H, Chen O, Ye Z, Zhang R, Hu H, Zhang N, Huang J, Liu W and Sun X: Inhalation of high concentrations of hydrogen ameliorates liver ischemia/reperfusion injury through A2A receptor mediated PI3K-Akt pathway. Biochem Pharmacol 130: 83-92, 2017

38. He Y, Shi JZ, Zhang RJ, Zhai DX, Zhang D, Yu CQ and Liu YH: Effects of hydrogen gas inhalation on endometriosis in rats. Reprod Sci 24: 324-331, 2017.

39. Peng Z, Chen W, Wang L, Ye Z, Gao S, Sun X and Guo Z: Inhalation of hydrogen gas ameliorates glyoxylate-induced calcium oxalate deposition and renal oxidative stress in mice. Int J Clin Exp Pathol 8: 2680-2689, 2015.
40. Chen JB, Kong XF, Lv YY, Qin SC, Sun XJ, Mu F, Lu TY and Xu KC: 'Real world survey' of hydrogen-controlled cancer: A follow-up report of 82 advanced cancer patients. Med Gas Res 9: 115, 2019.

41. Panchal SK and Brown L: Rodent models for metabolic syndrome research. J Biomed Biotechnol 2011: 351982, 2010.

42. Nakao A, Toyoda Y, Sharma P, Evans M and Guthrie N: Effectiveness of hydrogen rich water on antioxidant status of subjects with potential metabolic syndrome-an open label pilot study. J Clin Biochem Nutr 46: 140-149, 2010.

43. Hashimoto M, Katakura M, Nabika T, Tanabe Y, Hossain S, Tsuchikura S and Shido O: Effects of hydrogen-rich water on abnormalities in a SHR Cg-Lepr cp/NDmer rat-a metabolic syndrome rat model. Med Gas Res 1: 26, 2011.

44. Korovljev D, Trivic T, Drid P and Ostojic S: Molecular hydrogen affects body composition, metabolic profiles, and mitochondrial function in middle-aged overweight women. Ir J Med Sci 187: 85-89, 2018.

45. Zhai X, Chen X, Lu J, Zhang Y, Sun X Huang Q and Wang Q: Hydrogen-rich saline improves non-alcoholic fatty liver disease by alleviating oxidative stress and activating hepatic PPAR $\alpha$ and PPAR $\gamma$. Mol Med Rep 15: 1305-1312, 2017.

46. Panchal SK, Poudyal H, Iyer A, Nazer R, Alam MA, Diwan V, Kauter K, Sernia C, Campbell F, Ward L, et al: High-carbohydrate high-fat diet-induced metabolic syndrome and cardiovascular remodeling in rats. J Cardiovasc Pharmacol 57: 611-624, 2011.

47. Wang O, Liu J, Cheng Q, Guo X, Wang Y, Zhao L, Zhou F and Ji B: Effects of ferulic acid and $\gamma$-oryzanol on high-fat and high-fructose diet-induced metabolic syndrome in rats. PLoS One 10: e0118135, 2015

48. Tu LN, Showalter MR, Cajka T, Fan S, Pillai VV, Fiehn O and Selvaraj V: Metabolomic characteristics of cholesterol-induced non-obese nonalcoholic fatty liver disease in mice. Sci Rep 7: $6120,2017$.

49. YetukuriL,Katajamaa M,Medina-GomezG,Seppänen-Laakso T, Vidal-Puig A and Orešič M: Bioinformatics strategies for lipidomics analysis: characterization of obesity related hepatic steatosis. BMC Syst Biol 1: 12, 2007.

50. Sanders F, McNally B and Griffin JL: Blood triacylglycerols: A lipidomic window on diet and disease. Biochem Soc Tran 44: 638-644, 2016.

51. Hernández-Rodas MC, Valenzuela R, Echeverría F, Rincón-Cervera MA, Espinosa A, Illesca P, Muñoz P, Corbari A, Romero N, Gonzalez-Mañan D and Videla LA: Supplementation with docosahexaenoic acid and extra virgin olive oil prevents liver steatosis induced by a high-fFat diet in mice through PPAR- $\alpha$ and Nrf2 upregulation with concomitant SREBP-1c and NF-kB downregulation. Mol Nutr Food Res 61: 2017.

52. Echeverría F, Valenzuela R, Espinosa A, Bustamante A, Álvarez D, Gonzalez-Mañan D, Ortiz M, Soto-Alarcon SA and Videla LA: Reduction of high-fat diet-induced liver proinflammatory state by eicosapentaenoic acid plus hydroxytyrosol supplementation: Involvement of resolvins RvE1/2 and RvD1/2. J Nutr Biochem 63: 35-43, 2019.

53. Kawai D, Takaki A, Nakatsuka A, Wada J, Tamaki N, Yasunaka T, Koike K, Tsuzaki R, Matsumoto K, Miyake Y, et al: Hydrogen-rich water prevents progression of nonalcoholic steatohepatitis and accompanying hepatocarcinogenesis in mice. Hepatology 56: 912-921, 2012.

54. Iio A, Ito M, Itoh T, Terazawa R, Fujita Y, Nozawa Y, Ohsawa I, Ohno K and Ito M: Molecular hydrogen attenuates fatty acid uptake and lipid accumulation through downregulating CD36 expression in HepG2 cells. Med Gas Res 3: 6, 2013.

55. Ahmed MH and Byrne CD: Modulation of sterol regulatory element binding proteins (SREBPs) as potential treatments for non-alcoholic fatty liver disease (NAFLD). Drug Discov Today 12: 740-747, 2007.

56. Aragno M, Tomasinelli CE, Vercellinatto I, Catalano MG Collino M, Fantozzi R, Danni O and Boccuzzi G: SREBP-1c in nonalcoholic fatty liver disease induced by Western-type high-fat diet plus fructose in rats. Free Radical Bio Med 47: 1067-1074, 2009.

57. Valenzuela R and Videla LA: Impact of the co-administration of N-3 fatty acids and olive oil components in preclinical nonalcoholic fatty liver disease models: A mechanistic view. Nutrients 12: 499, 2020.

58. Paquette A, Wang D, Jankowski M, Gutkowska J and Lavoie JM: Effects of ovariectomy on PPAR alpha, SREBP-1c, and SCD-1 gene expression in the rat liver. Menopause 15: 1169-1175, 2008.

This work is licensed under a Creative Commons Attribution-NonCommercial-NoDerivatives 4.0 International (CC BY-NC-ND 4.0) License. 